\title{
Comparación de tres estrategias de tratamiento para la cefalea por sobreuso de medicación
}

\author{
Comparison of three treatment strategies for medication overuse headache
}

\section{Comentado de:}

Carlsen LN, et al. JAMA Neurol. 2020 Sep 1;77(9):1069-1078. PMID: $32453406^{1}$

\section{Objetivo}

Comparar tres estrategias de tratamiento para cefalea por sobreuso de medicamentos (CSM).

\section{Diseño, lugar y participantes}

Se realizó un ensayo clínico abierto, aleatorizado, de seis meses de seguimiento, en el Centro Danés de Cefalea (Glostrup, Dinamarca), un centro de tercer nivel, desde Octubre de 2016 a Junio de 2019.

Los participantes eran elegibles si presentaban los criterios diagnósticos de CSM de acuerdo a la Claificación Internacional de Trastornos de Cefalea, tercera edición (Versión Beta) (ICHD3 , por sus iniciales en inglés ${ }^{2}$ ):

A) Cefalea que ocurre $\geq 15$ días por mes en un paciente con un trastorno de cefalea preexistente;

B) Sobreuso regular por $>3$ meses de una o más drogas que pueden estar indicadas para la cefalea aguda o para el tratamiento sintomático del dolor de cabeza;

C) No cumple criterios para otro diagnóstico alternativo del ICHD-3.

Otros criterios de selección para participar en el estudio incluyeron: padecer como cefalea de base (primaria) una migraña o cefalea tensional (de acuerdo a los criterios ICHD-3); ser mayores de 18 años de edad; tener posibilidad de tratamiento ambulatorio y de completar un diario de cefaleas, entre otros. Como criterios de exclusión se destacaban la coexistencia de enfermedad severa (a nivel físico o psiquiátrico), la adicción al alcohol o drogas; el embarazo, la lactancia o la planificación de un embarazo en los siguientes 12 meses; la dificultad para proveer datos sobre su historial de salud (incluyendo la presencia de alguna barrera lingüística para el interrogatorio) y el uso de otros tratamientos preventivos para el dolor de cabeza.

\section{Intervención}

Se realizó la asignación aleatoria (1:1:1) de los participantes a uno de los tres tratamientos ambulatorios posibles:

- Grupo 1: Abstinencia + tratamiento preventivo desde el inicio
- Grupo 2: Tratamiento preventivo sin abstinencia

- Grupo 3: Abstinencia + tratamiento preventivo opcional, dos meses luego de la abstinencia.

La aleatorización fue por bloques de 9, y de tipo estratificada, con el fin de que los pacientes quedaran igualmente distribuidos en cada grupo acorde a su cefalea de base, ya que se consideró que ésta podría ser factor predictor de respuesta.

Los grupos de participantes a quienes se les indicó la abstinencia (grupo 1 y 3) recibieron entrenamiento individual en abstinencia y en CSM por parte de una enfermera especializada, seguida del retiro completo de analgésicos durante dos meses. El grupo de participantes asignados a la rama de tratamiento preventivo (grupo 2) recibió información general sobre la abstinencia en forma conjunta con la descripción del proyecto, sin limitación en el uso de medicación analgésica de corto plazo.

La medicación de rescate incluía la levomepromazina, la prometazina, la metoclopramida y la domperidona. Luego del periodo de abstinencia, los participantes de los grupos 1 y 2 podrían usar medicación de corto plazo hasta 9 días por mes (o 14 días por mes para los analgésicos simples solos); mientras que a quienes estaban asignados al grupo 3 se les ofreció tratamiento preventivo.

Los participandes de los grupos 1 y 2 recibieron fármacos preventivos seleccionados de acuerdo a las guías de práctica clínica del Centro Danés de Cefalea y a las comorbilidades de los pacientes.

\section{Medición de resultados principales}

El resultado primario fue el cambio en los días de cefalea por mes luego de seis meses de tratamiento.

Los resultados secundarios predefinidos fueron: cambio en los días de migraña por mes, uso de medicación de rescate, intensidad del dolor, número de respondedores, pacientes con reversión a cefalea episódica, y curación de CSM.

\section{Resultados}

Fueron incluidos en el estudio 120 pacientes, de los cuales 102 completaron un seguimiento a seis meses, con una edad media de 43,9 (desvío estándar 11,8) años y un total de 81 (79,4\%) mujeres. Los resultados principales se resumen en la Tabla 1.

Tabla 1. Cambios en los días mensuales de cefalea y proporción de curación de la cefalea por sobreuso de medicación (CSM) a los seis meses de seguimiento.

\begin{tabular}{|c|c|c|c|}
\hline Desenlaces & $\begin{array}{l}\text { Grupo 1: Abstinencia + Tratamiento } \\
\text { preventivo desde el inicio }(\mathrm{N}=31)\end{array}$ & $\begin{array}{l}\text { Grupo 2: Tratamiento } \\
\text { preventivo }(\mathrm{N}=35)\end{array}$ & $\begin{array}{l}\text { Grupo 3: Abstinencia } \\
(\mathrm{N}=36)\end{array}$ \\
\hline $\begin{array}{l}\text { Disminución en los días de cefalea por mes (intervalo } \\
\text { de confianza del } 95 \% \text { ) }\end{array}$ & $12,3(9,3$ a 15,3$)$ & $9,9(7,2$ a 12,6$)$ & $8,5(5,6$ a 11,5$)$ \\
\hline Proporción de pacientes con curación de la CSM & $96,8 \%$ & $74,3 \%$ & $88,9 \%$ \\
\hline
\end{tabular}


En cuanto a los cambios en los días de cefalea por mes, si bien podemos observar una diferencia en los valores centrales, los intervalos de confianza se superponen en los tres grupos, por lo que se interpreta que las diferencias no son significativas desde el punto de vista estadístico. Tampoco se encontraron diferencias en la reducción de los días de migraña por mes, en el uso de medicación de rescate o en la intensidad de la cefalea.

En relación a la curación de la CSM, los hallazgos muestran un $30 \%$ mayor probabilidad de curación en el grupo de tratamiento preventivo más abstinencia en comparación con el grupo de que recibió sólo tratamiento preventivo (riesgo relativo 1,3; intervalo de confianza del $95 \% 1,1$ a 1,6$)$

\section{Conclusión}

Las tres estrategias de tratamiento fueron efectivas, pero la estrategia de abstinencia más medicación preventiva desde el inicio, es la más recomendada para el tratamiento de la CSM.

Conflicto de interés de los autores: Varios autores del estudio reportaron haber recibido financiamiento de entidades públicas y privadas durante la conducción de este estudio, por fuera de él o en forma previa, descritas en detalle en el artículo original.

\section{Comentario}

La cefalea por sobreuso de medicación, también conocida como cefalea por rebote de analgésicos, cefalea inducida por analgésicos y cefalea por mal uso de analgésicos ${ }^{3}$, se define en la actualidad como aquella que ocurre durante 15 o más días por mes en un paciente con una cefalea primaria preexistente y que se desarrolla como consecuencia del sobreuso regular de medicación para la cefalea aguda o sintomática (10 o más días por mes para ergotamina, triptanes u opioides, o alguna combinación de estos analgésicos con analgésicos comunes, o al menos 15 días por mes de consumo de analgésicos comunes, como el paracetamol, la aspirina o los antiinflamatorios no esteroideos) por más de tres meses ${ }^{4}$. Esta definición es consistente con la empleada en el estudio resumido para el reclutamiento de los sujetos. Si bien la prevalencia de este problema de salud es baja en la población general (1 a $2 \%$ ), puede llegar al $50 \%$ en los centros especializados ${ }^{3}$.

Este es el primer ensayo aleatorizado en comparar distintas estrategias de tratamiento de la $\mathrm{CSM}^{5}$. Sin embargo, no está exento de limitaciones metodológicas, por lo que la confianza en la evidencia que aporta sobre las estrategias terapéuticas de esta entidad sigue siendo baja. Una de ellas es que no se presenta un grupo control (placebo) contra el cual comparar cada una de las tres estrategias de tratamiento, lo que no permite establecer la efectividad absoluta de cada intervención. Por otro lado, si bien los autores consideran que la tasa de abandono estuvo distribuida homogéneamente en los tres grupos, se observa que la proporción de abandono fue sensiblemente mayor en el grupo $1(22,5 \%)$ frente a los grupos $2(12,5 \%)$ y 3 $(10 \%)$. Por este motivo, la decisión de no realizar un análisis por intención de tratar basada en un potencial riesgo de sesgo por pérdida de pacientes bajo es cuestionable.

Adicionalmente, los fármacos utilizados en el estudio son diferentes a los recomendados por las guías de práctica clínica actuales como estrategia de tratamiento para la $\mathrm{CSM}^{6}$. Esta observación, sumado a que el ensayo fue realizado en un centro de tercer nivel, disminuye la validez externa del estudio para la atención primaria de la salud.

Es importante destacar que, en casos de cefalea primaria compleja, por ejemplo una migraña severa, la estrategia de indicar la abstinencia completa de analgésicos sería difícil de implementar en la práctica, por lo que la guía NICE ya recomendaba la contemplación de indicar tratamiento profiláctico de la cefalea primaria, antes de esta publicación 6 .

\section{Conclusiones de la comentadora}

Si bien las tres estrategias evaluadas en este estudio parecen tener eficacia en la mejoría de los síntomas de los pacientes que padecen CSM, la estrategia que combina la abstinencia con la profilaxis podría ser la más recomendable, siempre que sean contempladas características y las preferencias de cada paciente en forma individual.

Catalina Morán [ Servicio de Medicina Familiar y Comunitaria, Hospital Italiano de Buenos Aires. catalina.moran@hospitalitaliano.org.ar ]

Morán C. Comparación de tres estrategias de tratamiento para la cefalea por sobreuso de medicación. Evid Actual Pract Ambul. $2021 ; 24(2): e 002125$. Comentado de: Carlsen LN, et al. Comparison of 3 Treatment Strategies for Medication Overuse Headache: A Randomized Clinical Trial. JAMA Neurol. 2020 Sep 1;77(9):1069-1078. PMID: 32453406

\section{Referencias}

1. Carlsen LN, Munksgaard SB, Nielsen M, et al. Comparison of 3 Treatment Strategies for Medication Overuse Headache A Randomized Clinical Trial. JAMA Neurol. 2020;77(9):1069-1078. Available from: doi:10.1001/jamaneurol.2020.1179.

2. Headache Classification Committee of the International Headache Society (IHS). The International Classification of Headache Disorders, 3rd edition (beta version). Cephalalgia. 2013;33(9):629-808. Available from: 10.1177/0333102413485658.

3. Garza I, Schwedt TJ. Medication overuse headache: Etiology, clinical features, and diagnosis. In: Swanson JW, editor. UpToDate. Wolters Kluwer; 2019.

4. Headache Classification Committee of the International Headache Society (IHS) The International Classification of Headache Disorders, 3rd edition. 2018;38(1):1-211. Available from: 10.1177/0333102417738202.

5. Chiang CC, Schwedt TJ, Wang SJ, et al. Treatment of medication-overuse headache: A systematic review. Cephalalgia. 2014;36(4):371-86. Available from: 0.1177/0333102415593088.

6. NICE. Diagnosis and management of headaches in young people and adults. (Clinical Guideline 150 Headaches.); 2012. Available from: https: //www.nice.org.uk/guidance/cg150. 